\title{
Measured Electrical Properties of Snow and Glacial Ice
}

\author{
A. D. Watt and E. L. Maxwell
}

(January 22, 1960)

\begin{abstract}
The electrical properties of snow and glacial ice near $0^{\circ} \mathrm{C}$ have been observed over the frequency range from 20 cycles per second to 200 kilocycles per second. In general, the conductivity of snow and glacial ice is found to be much higher than that for pure ice. This is particularly so at frequencies below 2 kilocycles per second.

The magnitude of the complex conductivity for glacial ice appears to increase with temperature at frequencies below 200 cycles per second and to decrease with temperature above this frequency.
\end{abstract}

\section{Introduction}

The electrical properties (i.e., conductivity and permittivity) of snow and ice can have an appreciable effect upon the propagation of electromagnetic waves over such media. Similarly, the characteristics of ground-based antennas may be severely modified $[1,2] .^{3} \quad$ Although the properties of pure ice as measured in the laboratory are relatively well known, the physical characteristics and formation of snow and glacial ice are different, and thus it is important that their properties be measured under actual field conditions. Considerable dispersion exists in the electrical properties of ice throughout the audiofrequency range, and in order to permit measurements of the electrical properties of snow and glacial ice in their natural state, two independent methods have been employed over the frequency range from $20 \mathrm{cps}$ to $200 \mathrm{kc}$. These are described in the following section and the resulting electrical properties presented.

\section{Measurement Methods and Nomenclature}

The bridge and substitution method shown in figure 1 permits observations to be made on the conductivity and permittivity (dielectric constant) of a lossy dielectric. Numerous forms of electrodes have been employed including parallel plates and the parallel rods shown. The parallel rods have the great advantage of permitting their insertion into the medium by the simple expedient of drilling two holes with an ice auger. This method produces a minimum amount of disturbance in the medium, and when the experiment is carefully conducted the effects of fringe capacity etc., are greatly minimized. This method suffers from one primary deficiency in that the electrodes must fit very closely in the ice, and there is always the question of possible errors in

${ }_{1}^{1}$ Contribution from Central Radio Propagation Laboratory, National Bureau of Standards, Boulder, Colo.

2 The studies contained in this paper were sponsored by the Office of Naval Research under Contract No. NR 371-291.

3 Figures in brackets indicate the literature references at the end of this paper.

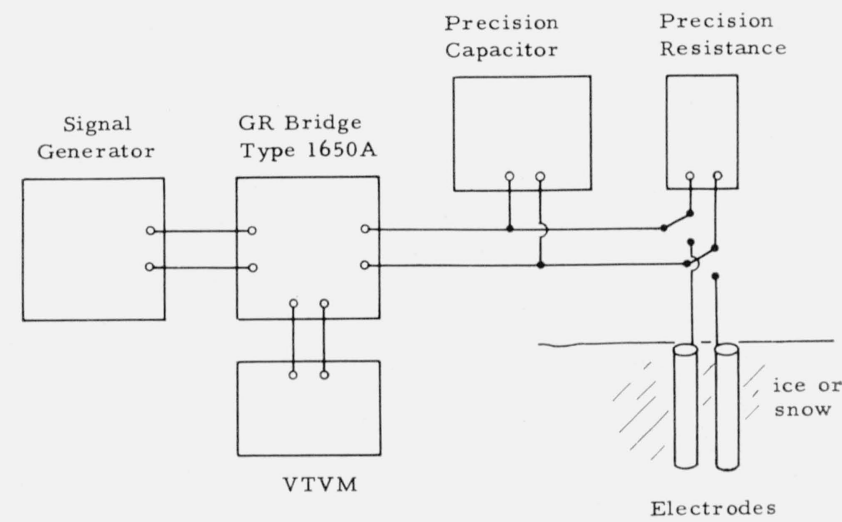

Figure 1. Bridge and substitution method for measuring conductivity and dielectric constant.

the determination of conductivity and permittivity due to interface or surface-layer effects on the electrodes themselves.

The other method employs two pairs of electrodes. It is described generally by Wait [3] and has been used in measuring ground conductivity in the audiofrequency region. The use of four electrodes permits a determination of the magnitude of the complex conductivity $|\bar{\sigma}|$ to be made in such a manner that surface-layer effects [4] at the electrodes are eliminated. At least three basic arrangements of electrodes were employed during these measurements including the Wenner, Eltran, and right-angle arrays. The Eltran arrangement shown in figure 2 proved to be the convenient form to use since it permits a ready changing of electrode spacings, and accurate results can be obtained over a relatively wide range of electrode spacings provided that the precaution of elevating the wire several feet above the surface of the snow or ice is taken. If the wires connecting the electrodes were not elevated, the large dielectric constant of the snow and the rather high terminal resistance resulted in a nonuniform distribution of current along the wire. This, of course, invalidates the relations employed in calculating $|\bar{\sigma}|$ based upon a model where the current and potential electrodes 
are discrete points. At very short spacings, the results with all three methods were similar.

It is important that the vacuum-tube voltmeter across the potential electrodes have a very high input impedance and that the voltmeter stake impedances be made as low as possible by pouring salt or salt water around electrodes placed in the ice so that the voltage indicated is equal to the true potential difference between the potential electrodes. These precautions are necessary since the stake impedances are effectively in series with the potential source whose voltage is desired. In some cases, it may be difficult to reduce the stake impedances below $0.5 \mathrm{meg}$ and corrections of the voltmeter readings may be necessary [4].

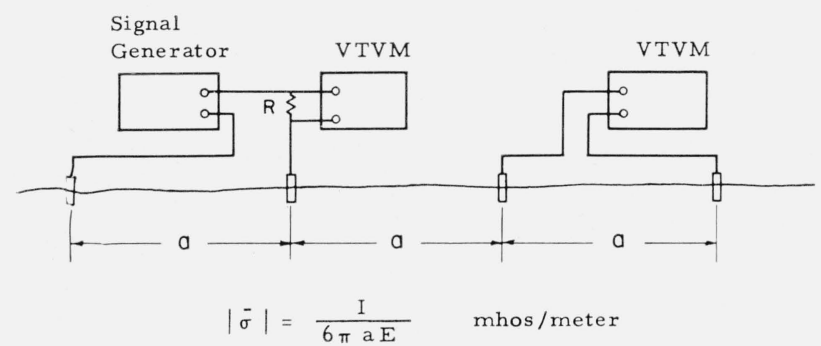

Figure 2. Four electrode [ELTRAN] array for measuring the magnitude of the complex conductivity.

The signal generator should be capable of producing relatively high voltages so that sufficient current can be induced into the current electrodes. This makes the voltage produced at the potential electrodes appreciably greater than the background noise caused by atmospheric noise and earth-current fields. Signal generators with outputs of $1 \mathrm{v}$ have been employed for spacings up to $20 \mathrm{ft}$ while beyond this it has been found necessary to employ an additional power amplifier with a capability of supplying $300 \mathrm{v}$ into $6,000 \mathrm{ohms}$. This arrangement has permitted electrode spacings of up to $1,000 \mathrm{ft}$ provided that some filtering is employed at the vacuum-tube voltmeter to eliminate the fields produced by VLF transmitting stations.

The four-electrode method using the instrumentation shown eliminates the effects of surface phenomena at the electrodes but does not give the magnitude of the conductivity and permittivity independently. ${ }^{4}$ The magnitude of the complex conductivity employed by this means can, however, be compared with that obtained by the bridge substitution method as a check to see whether or not surface phenomena at the electrodes in the bridge measurements was great enough to cause an error in the observations.

The physical characteristics of the electrodes, used in the bridge method, and the relation between the equivalent circuit and the basic properties of the dielectric material are shown in figure 3. The two basic physical properties to be measured are the conductivity $\sigma$ expressed in (mhos/meter), and the permittivity (dielectric constant), $\epsilon$, expressed in

${ }^{4}$ This can be accomplished if the relative phase batween current $I$ and voltage $V$ is measured [4].

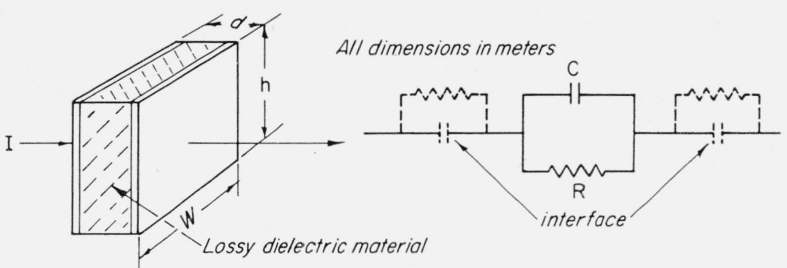

LIST OF SYMBOLS

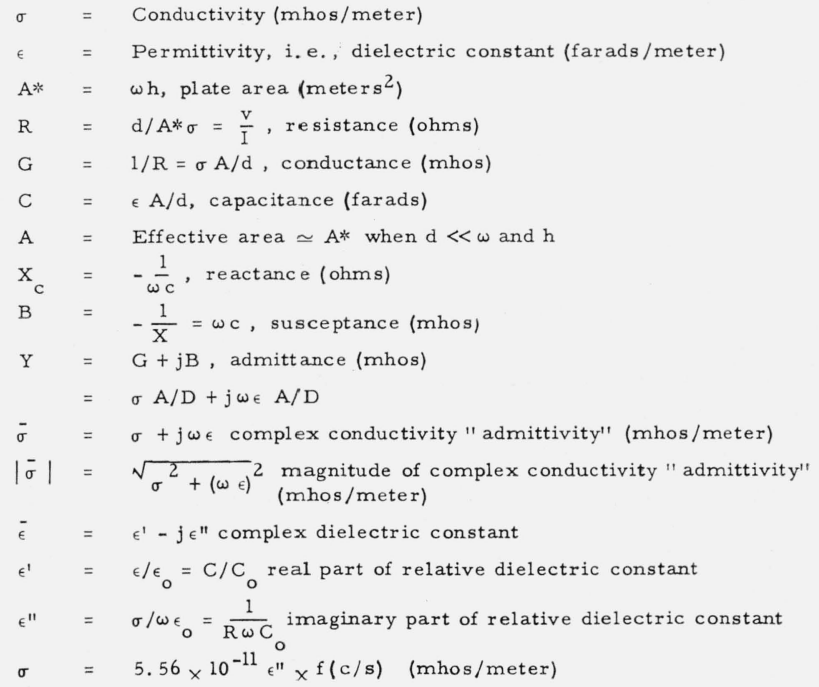

FiguRE 3. Lossy dielectric capacitor and equivalent circuit.

(farads/meter). When the plates or rods are immersed in an effectively infinite medium, it is necessary to use the effective area to separation ratio $A / d$ rather than the ratio of physical values $A^{*} / d$ shown in the illustration.

It is evident that

$$
\sigma=\frac{1}{R A / d}
$$

and that

$$
\epsilon=\frac{C}{A / d}
$$

where $\sigma$ is the conductivity in mhos/meter, $R$ is the equivalent parallel resistance in ohms, $A$ is the effective area in square meters, $d$ is the effective separation in meters, $\epsilon$ is the permittivity of the substance in farads/meter, and $C$ is the capacity in farads. Since the ratio $A / d$ may influence the interface effect, the value of this ratio is given for each electrode arrangement employed. Although $A / d$ can be calculated with fairly good accuracy, the value determined experimentally from the air capacity of the electrodes is used in all the calculations of this paper. Most of the nomenclature employed is now well standardized [4]. The complex conductivity, $\bar{\sigma}$, may not be well known and the possibility of employing "admittivity" in future work is suggested. It should be emphasized that the quantity obtained by the four-electrode method is $|\bar{\sigma}|$ the magnitude of 
the "admittivity" rather than the conductivity $\sigma$ which is the dominant term in most ground measurements.

\section{Properties of Pure Ice}

The electrical and physical properties of ice have been discussed in considerable detail by Dorsey [5]. He presents tabulations of permittivity and conductivity as reported by numerous observers. A more recent paper by Auty and Cole [6] has analyzed the lack of close agreement between measured dielectric constants of ice and has found that in general they appear to be attributable to the formation of voids or cracks in the samples being measured.

For a material with a single relaxation frequency the simple theory of dielectric relaxation predicts a complex dielectric constant $\bar{\epsilon}$

$$
\bar{\epsilon}=\epsilon^{\prime}-i \epsilon^{\prime \prime}=\epsilon_{\infty}+\left(\epsilon_{d c}-\epsilon_{\infty}\right) /\left(1+i f / f_{c}\right)
$$

where the time factor is $e^{i \omega t}, \epsilon_{d c}$, and $\epsilon_{\infty}$ are the socalled equilibrium and limiting high-frequency values of relative dielectric constant, $f$ is the frequency at which the electrical properties are being measured, and $f_{c}$ is defined as the relaxation frequency which is equal to $1 / \tau$, where $\tau$ is the relaxation time. The complex plane presentation of $\bar{\epsilon}$ forms semicircles as shown in figure 4. This type of presentation is known as the Argand diagram. Auty and Cole [6]

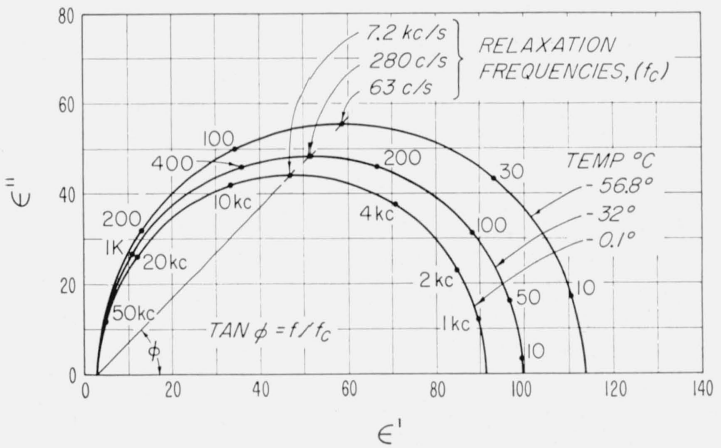

FIGURE 4. Electrical properties of pure ice. From Auty and Cole.

with carefully designed equipment in experimental procedures found that pure ice does in fact adhere very closely to the form predicted by this simple theory, and they were able to obtain results with a high degree of repeatability. From their data we have obtained the relaxation frequency $f_{c}$ as a function of temperature as shown in figure 5 . The high-frequency dielectric constant $\epsilon_{\infty}$ was found to be very close to 3 for all temperatures ranging from $-0.1^{\circ}$ to $-65.8^{\circ} \mathrm{C}$; and $\epsilon_{d c}$, also shown in figure 5 , has values ranging from approximately 90 to 114 over the temperature range indicated. The value of $\epsilon$ corresponding to the relaxation frequency, $\epsilon_{c}$, permits a rapid construction of Argand diagrams since this point represents the center of the circular diagram. It is obvious that the frequency at the

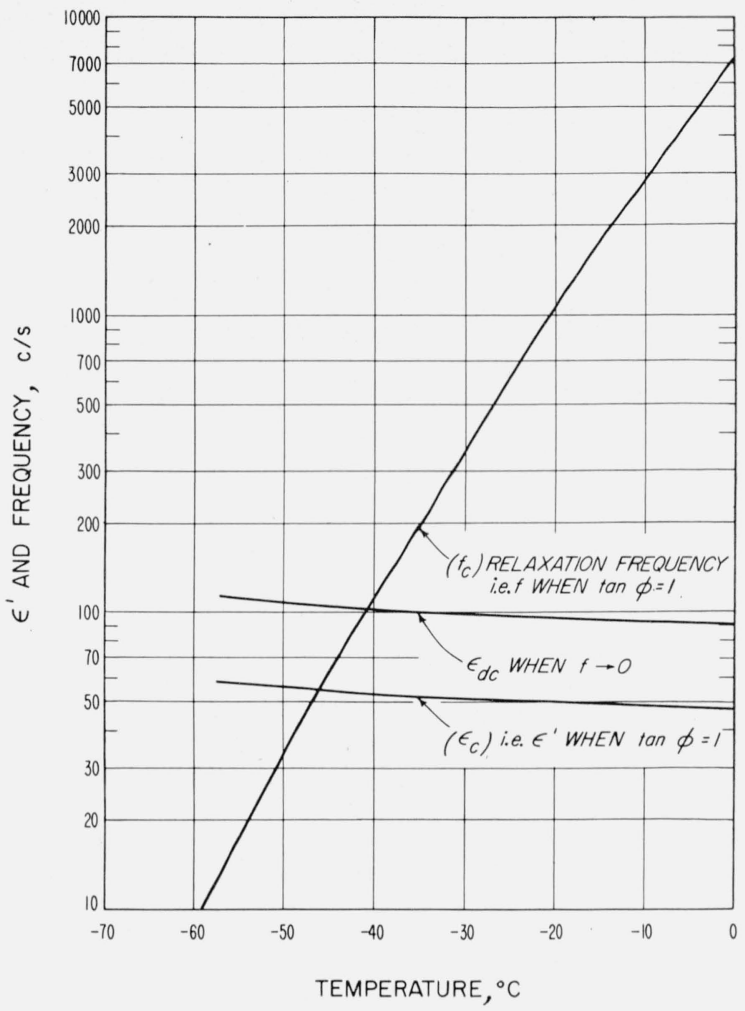

Figure 5. Pure ice-frequency and relative permittivity when $\tan \phi=1$ versus temperature.

From Auty and Cole.

top of the semicircular diagram will be the relaxation frequency. Frequencies at other points on this diagram can readily be obtained by the relationship

$$
\tan \phi=f / f_{c}
$$

where $\phi$ is the angle indicated in figure 4 . The relative dielectric constant $\epsilon^{\prime}=\epsilon / \epsilon_{0}$ can be readily obtained from diagrams such as figure 4 and the results presented as a function of frequency for various temperatures as shown in figure 6.

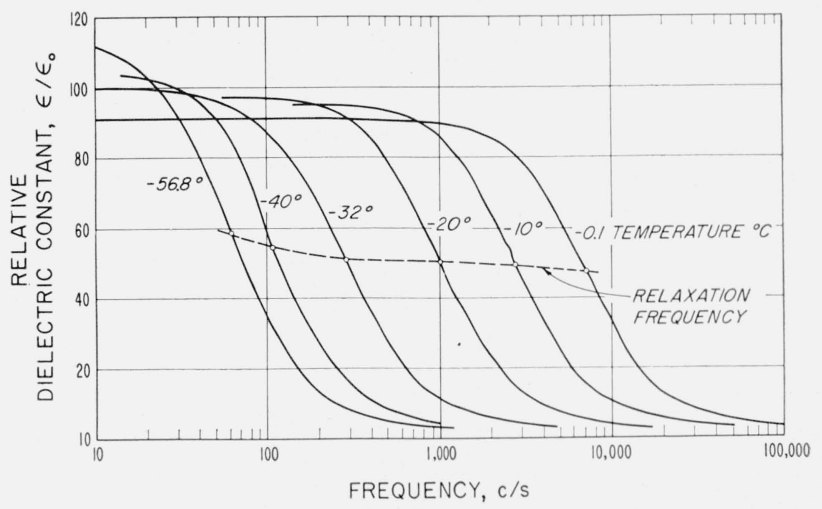

FIGURE 6. Relative dielectric constant of pure ice. Calculation based on Auty and Cole. 
It is interesting to note that the equilibrium relative dielectric constant $\epsilon_{d c}$ increases with decrease in temperature. This same general increase in $\epsilon_{d c}$ has been predicted theoretically by Powles [7]. The actual magnitude, however, is either below or somewhat above the values obtained experimentally depending upon the type of theoretical model employed in the calculations.

The conductivity of pure ice can be obtained from the Argand diagram of figure 4 by means of the relationship

$$
\sigma=\epsilon^{\prime \prime} \omega \epsilon_{0}
$$

which can also be written as

$$
\sigma=5.56 \times 10^{-11} \epsilon^{\prime \prime} f
$$

where $f$ is the frequency in cycles per second and $\sigma$ is the conductivity in mhos/meter. The results of these calculations, shown in figure 7 , indicate that for a given temperature and at frequencies below the relaxation frequency that the conductivity is directly proportional to the square of the frequency.

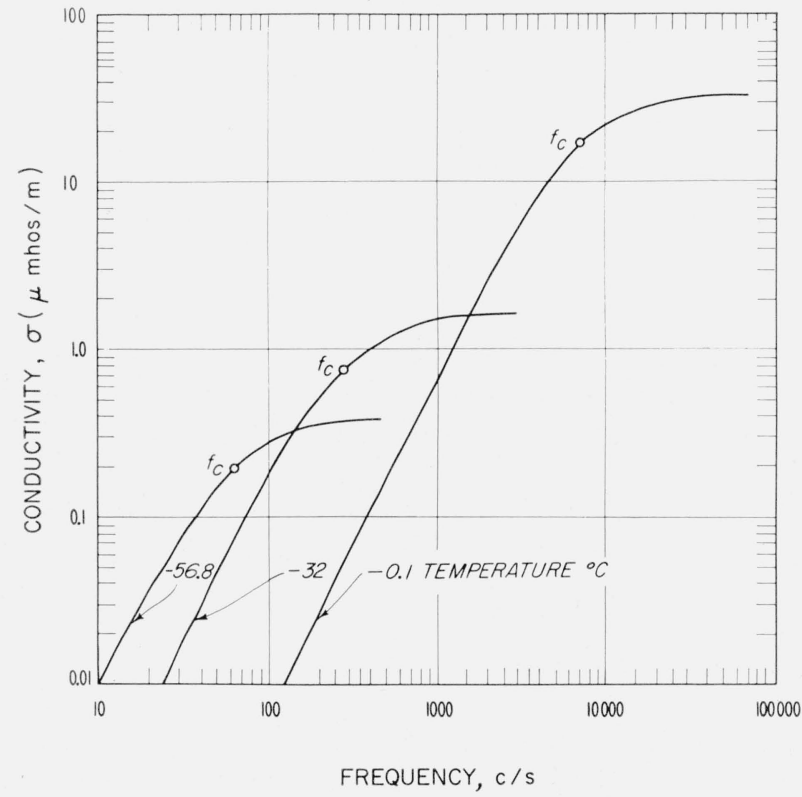

Figure 7. Conductivity of pure ice.

Calculations based on Auty and Cole.

\section{Experimental Results}

\subsection{Snow Drifts in Colorado}

The properties of drifted snow were measured at two locations in the mountains of Colorado. The Argand diagrams resulting are shown in figure 8 where it is obvious that the characteristics are different from those of pure ice. Four electrode methods of measuring the absolute magnitude of the complex conductivity are also employed and a comparison of results is shown in figure 9, where it is seen that relatively good agreement exists between the two types of measurements. The type of

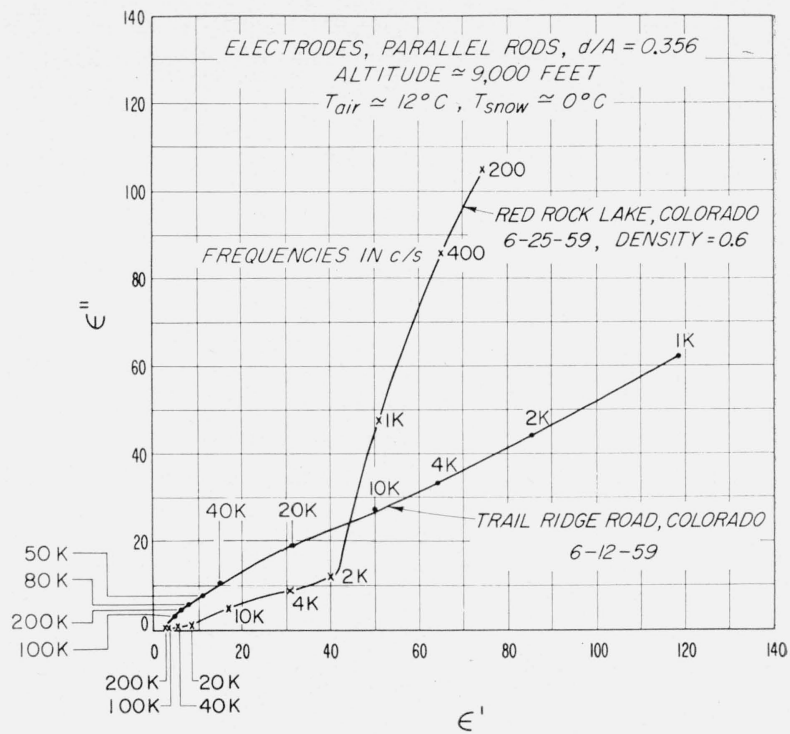

Figure 8. Electrical properties of spring drift snow. Compact and wet.

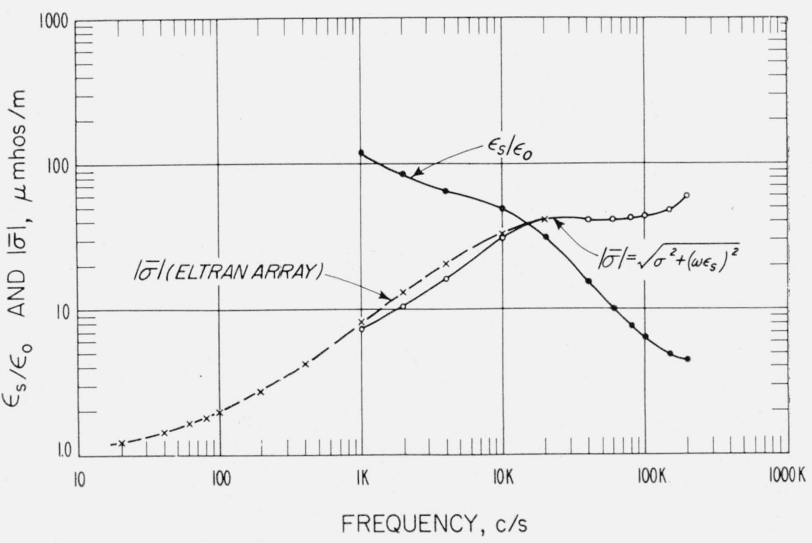

Figure 9. Electrical properties of wet snow.

Trail Ridge Road, Colorado, June 12, 1959.

departures from pure ice are in general agreement with observations made by Kuroiwa [8] and Devaney [9]. It should be pointed out that the rather large values of $\epsilon^{\prime \prime}$ which result from an increase in conductivity are probably due to the presence of impurities in the snow and possible interface (overvoltage) effects at the particles [4]. The effect of impurities was verified by Kuroiwa by means of chemical analysis of the snow and by an experiment in which he produced hoarfrost crystals from chemically pure water and found that the Argand diagram was a semicircle as observed with pure ice although the dielectric constant was, of course, much lower because of the decreased density.

\subsection{Athabasca Glacier, Columbia Icefields, Canada}

Observations made on the Athabasca glacier near Jasper in Alberta, Canada, produced the Argand diagram shown in figure 10 . It is seen from this 


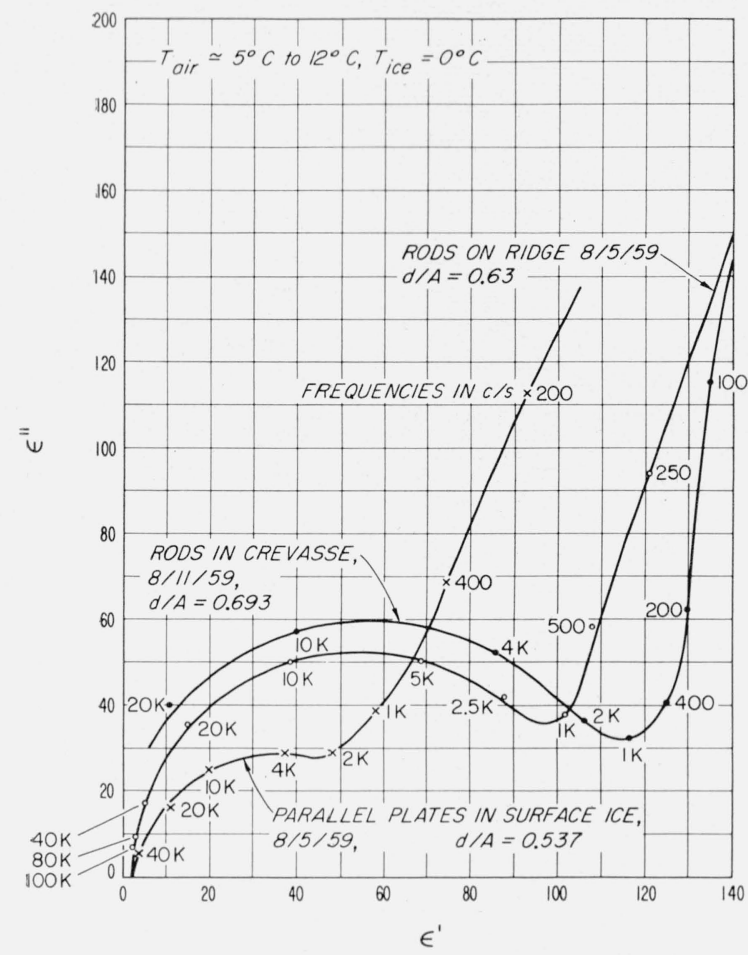

Figure 10. Electrical properties of glacial ice. Athabasca glacier, Alberta, Canada.

figure that the general circular form expected for pure ice having a simple dielectric relaxation is obtained for frequencies down to approximately $2 \mathrm{kc}$. Below this the $\epsilon^{\prime \prime}$ values increase quite rapidly indicating an appreciable amount of conductivity.

It should be noted that the radius of these Argand diagrams is greater than that predicted for $0^{\circ} \mathrm{C}$ pure ice from figure 4 . This general type of departure was also observed by Auty and Cole [6] who explained the discrepancy on the basis of electrode interface effects. They indicate that this effect disappears at larger electrode spacings which, for the equipment they employed, would be a $d / A$ ratio of 29 . The smallest ratio of $d / A$ which they employed was in the order of 3.3 . In our experiments $d / A$ varied from 0.36 to 0.69 . A comparison of the magnitude of the complex conductivity as obtained by bridge and four-electrode Eltran measurements, figure 11, shows close agreement. This may be due to the fact that the rods were surrounded by a very thin interface of water. These sets of observations were obtained in the same general area on the glacier and would indicate that the large observed values of $\epsilon^{\prime}$ and $\epsilon^{\prime \prime}$ are probably real. It should be pointed out that some dispersion was found between various observations in different areas on the glacier due possibly to cracks and crevasses.

The parallel plate observations of figure 10 were made near the surface of the ice which was rather porous and wet at the time of measurement. The rods on the ridge were placed in the same general area as the parallel plate measurements, but being $4 \mathrm{ft}$ long are expected to give results typical for ice several

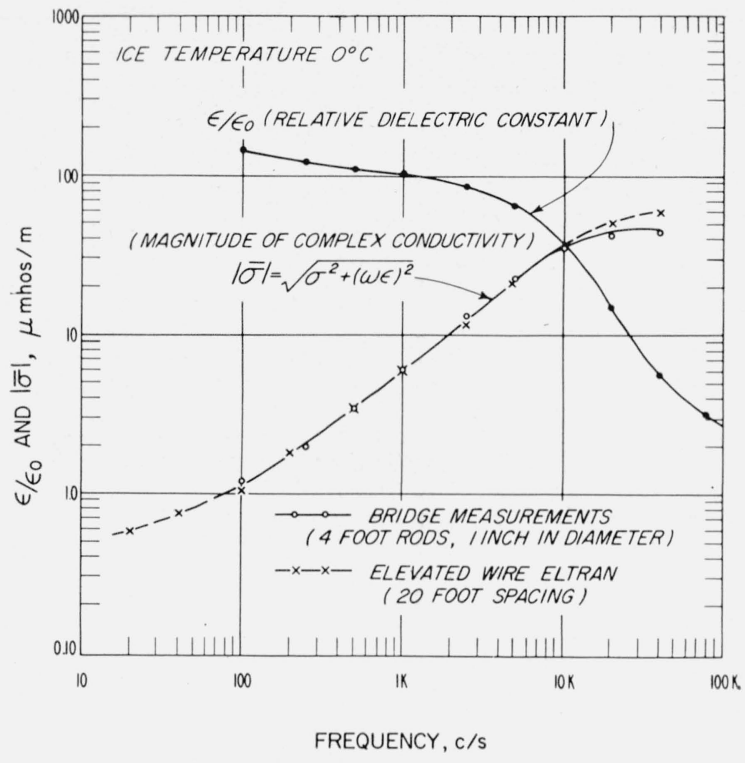

FIgURE 11. Electrical properties of glacial ice. A thabasca glacier, Alberta, Canada, Aug. 5, 1959.

feet below the surface which was observed to be much more solid than the surface ice. The third curve for rods in a crevasse represents measurements made some $30 \mathrm{ft}$ below the surface of the glacier where the ice was less disturbed by wind action, and in addition, it was expected to be slightly colder than the surface ice. This reduction in temperature with depth is indicated by the fact that rods in or near the surface caused melting around them while rods a hundred feet or so below the surface would freeze in place.

Figure 12 shows $|\bar{\sigma}|$ as a function of frequency for three different electrode spacings on the Athabasca

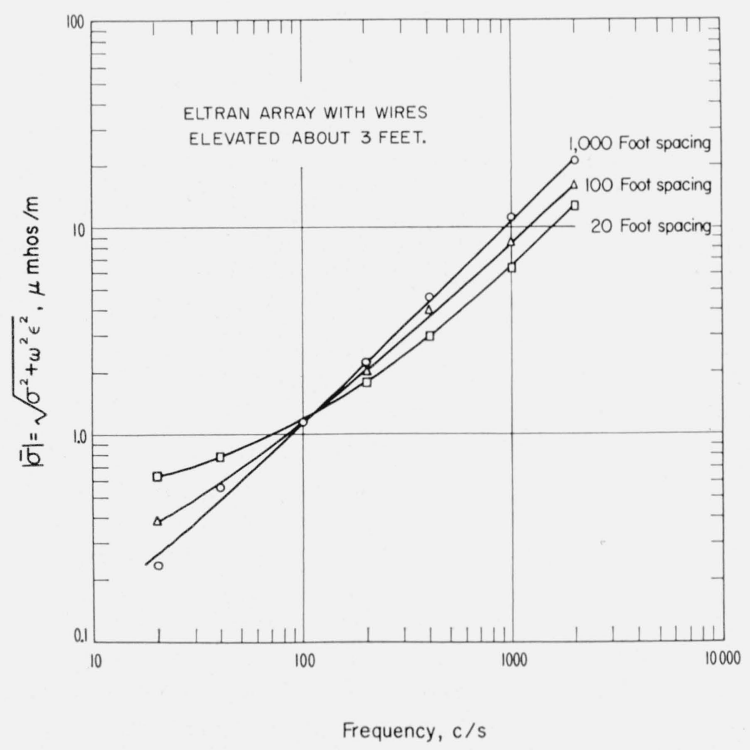

Figure 12. Magnitude of complex conductivity vs. frequency at three electrode spacings.

Athabasca glacier, Alberta, Canada, Aug. 1959. 
glacier. It is interesting to note the reversal in magnitude at the $100-$ cps region. Similar data is shown in figure 13 where $|\bar{\sigma}|$ is plotted as a function of electrode spacing at different frequencies. It is obvious from these results that the properties of the glacier change with depth and that this change has an inverse effect at frequencies above and below the 100-cps region.

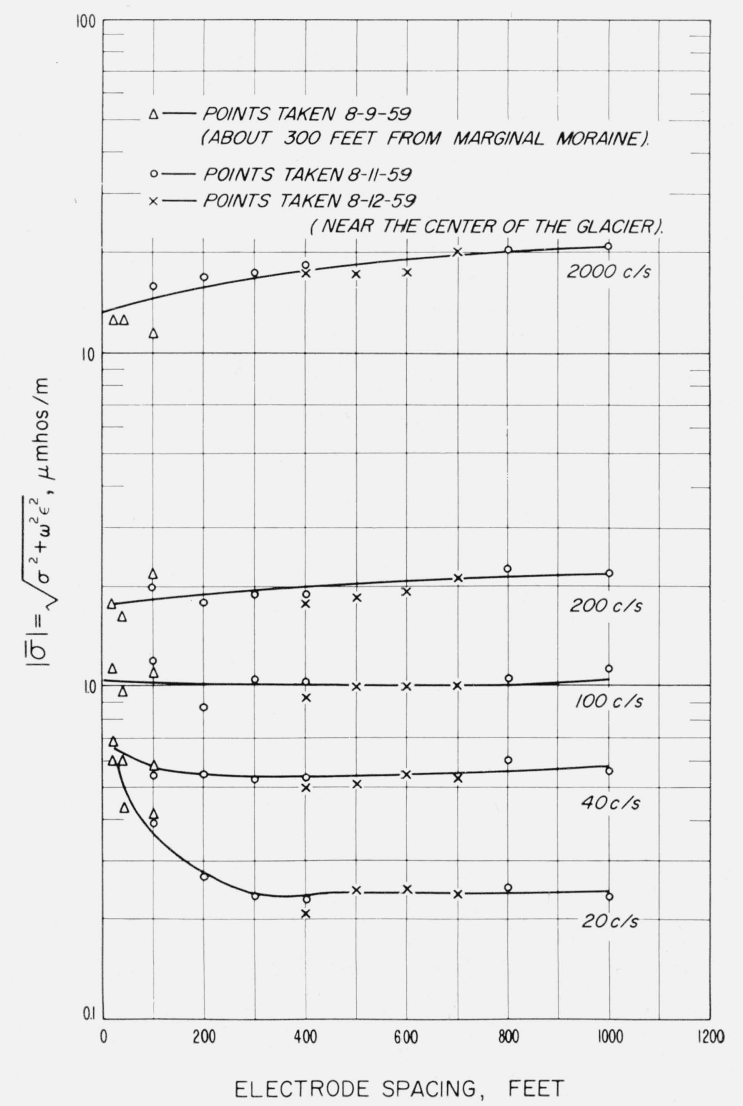

FIGURE 13. Variations of magnitude of complex conductivity Athabasca glacier, Alberta, Canada.

The magnitude of the complex conductivity, $|\bar{\sigma}|$, obtained by means of the bridge measurements is shown in figure 14, and it is interesting to observe that on the surface $|\bar{\sigma}|$ is greater below $200 \mathrm{cps}$ and less above this frequency than is found for the subsurface measurements. This general behavior is in agreement with the observations of figures 12 and 13 when the variation of effective penetration with stake spacing is considered. This effective physical depth for the various electrode spacings is discussed in considerable detail by Wait [3].

It is instructive to observe the manner in which the conductivity, admittivity, and loss tangent vary as a function of frequency. A typical example taken from the Athabasca glacier for $0^{\circ} \mathrm{C}$ glacial ice is shown in figure 15 where it can be seen that the loss tangent increases below $1 \mathrm{kc}$ rather than continuing to decrease as it does for pure ice.

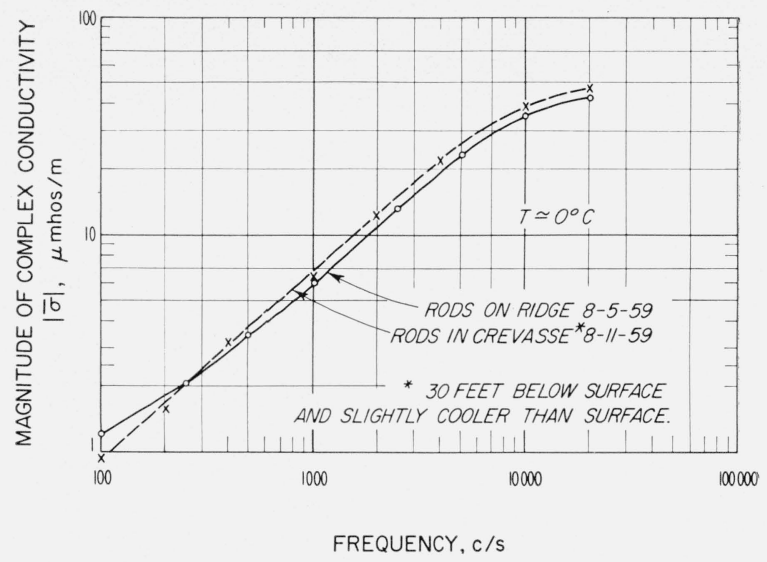

FiguRE 14. Magnitude of complex conductivity for surface and subsurface ice.

Athabasca glacier, Alberta, Canada.

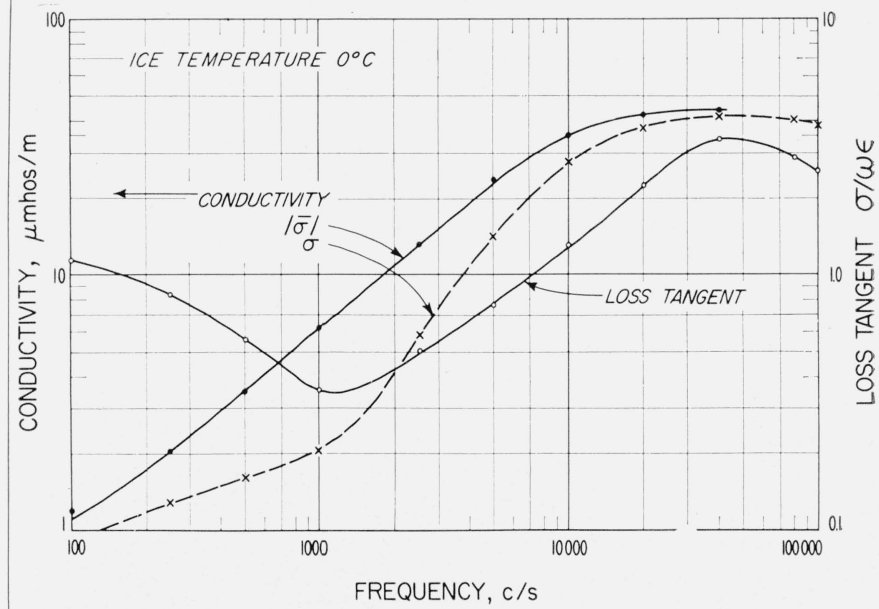

Figure 15. Electrical properties of glacial ice. Athabasca glacier, Alberta, Canada, Aug. 5, 1959.

\section{Conclusions}

It is apparent from our limited observations that the electrical properties of snow and glacial ice are appreciably different than those of pure ice. The greatest difference appears in the region below $2 \mathrm{kc}$. The conductivity, $\sigma$, of glacial ice appears to be approaching a constant in the vicinity of 0.5 to $3 \mu$ mhos $/ \mathrm{m}$ for frequencies of $200 \mathrm{cps}$ and lower. For pure ice, $\sigma$ appears to be continuing to decrease with decreasing frequency and at $100 \mathrm{cps}, \sigma \cong 0.01 \mu$ $\mathrm{mhos} / \mathrm{m}$ at $-0.1^{\circ} \mathrm{C}$. Typical values of $\sigma$ at $20 \mathrm{kc}$ are $30 \mu \mathrm{mhos} / \mathrm{m}$ for pure ice, and $36 \mu \mathrm{mhos} / \mathrm{m}$ for the ice of the Athabasca glacier.

The close agreement between the values of complex conductivity magnitude obtained with the bridge measurements and four electrode methods would appear to indicate that electrode surface effects have not greatly influenced the results reported here. For future bridge measurements it 
does, however, appear desirable to employ several rod spacings including $d / A$ ratios up to 10 or more. For general propagation studies and wave-tilt calculations, the values of $\sigma$ and $\epsilon$ given for ice should in most cases be used in the stratified earth formulas of Wait [10] rather than in the simple relation for an infinitely deep layer. Typical values of $\sigma$ for the material below the ice may vary from 0.1 to 10 millimhos $/ \mathrm{m}$. Surface measurements near the Athabasca glacier yielded values essentially independent of frequency of $\sigma \sim 2$ millimhos/m for soil in the creek beds, and $\sim 0.3$ millimhos $/ \mathrm{m}$ for a rocky ridge.

The authors acknowledge the assistance obtained from: J. R. Wait, for many valuable suggestions during the course of this work; E. C. Bolton and R. W. Plush, for assistance in preparation of equipment and obtaining data; Henri Vetter and D. R. Watt, for assisting in the measurements on the Athabasca Glacier: G. D. Garland, whose interest made the measurements on the glacier possible; F. J. Weyl, for his interest and encouragement during the course of this work; and Mrs. Winifred Mau, for her assistance during the preparation of this manuscript.

\section{References}

[1] J. R. Wait and A. M. Conda, Pattern of an antenna on a curved lossy surface, IRE Trans. PGAP AP-6, 348 (1958)

[2] A. D. Watt, E. L. Maxwell, and E. H. Whelan, Lowfrequency propagation paths in Arctic areas, J. Research NBS 63D, 99 (1959).

[3] J. R. Wait and A. M. Conda, On the measurement of ground conductivity at VLF, IRE Trans. PGAP AP-6, 273 (1958).

[4] J. R. Wait (Ed.), Overvoltage research and geophysical applications (Pergamon Press, New York, N.Y., 1959).

[5] N. E. Dorsey, Properties of ordinary water-substance (Reinhold Publishing Corp., New York, N.Y., 1940).

[6] R. P. Auty and R. H. Cole, Dielectric properties of ice and solid $\mathrm{D}_{2} \mathrm{O}$, J. Chem. Phys. 20, 1309 (1952).

[7] J. G. Powles, A calculation of the static dielectric constant of ice, J. Chem. Phys. 20, 1302 (1952).

[8] D. Kuroiwa, The dielectric property of snow (Inst. Low Temp. Sci., Hokkaido Univ., Soygcoro, Japan) Proc. IGU, Rome (1954).

[9] T. E. Devaney (private communication).

[10] J. R. Wait, Propagation of radio waves over stratified ground, Geophysies 18, 416 (1953).

(Paper 64D4-69) 